\title{
BMJ Open Quality improvement strategies to optimise transition of patients with heart failure to independent living: protocol for a scoping review
}

\author{
Monika Kastner, ${ }^{1}$ Erin Lillie, ${ }^{1}$ Huda Ashoor, ${ }^{1}$ Laure Perrier, ${ }^{1}$ Roberta Cardoso, ${ }^{1}$ \\ Sharon Straus, ${ }^{1}$ Douglas S Lee ${ }^{2,3}$
}

To cite: Kastner M, Lillie E, Ashoor $\mathrm{H}$, et al. Quality improvement strategies to optimise transition of patients with heart failure to independent living: protocol for a scoping review. BMJ Open 2014;4:e005711. doi:10.1136/bmjopen-2014005711

- Prepublication history and additional material is available. To view please visit the journal (http://dx.doi.org/ 10.1136/bmjopen-2014005711).

Received 16 May 2014 Revised 24 October 2014 Accepted 6 November 2014

CrossMark

\begin{abstract}
${ }^{1}$ Li KaShing Knowledge Institute, St Michael's Hospital, Toronto, Ontario, Canada

${ }^{2}$ Peter Munk Cardiac Centre and the Joint Department of Medical Imaging of University Health Network, University of Toronto, Toronto, Ontario, Canada

${ }^{3}$ Institute for Clinical Evaluative Sciences, Toronto, Ontario, Canada
\end{abstract}

Correspondence to Dr Monika Kastner; monika.kastner@utoronto.ca

\begin{abstract}
Introduction: Heart failure (HF) is a leading reason for hospitalisation and readmissions to hospital,

particularly among individuals older than 65 years of age. The prognosis of patients with $\mathrm{HF}$ is grim, with high rates of mortality risk and hospital readmissions. The transition period early after hospital discharge represents a window of opportunity to positively influence patient outcomes using quality improvement (Ql) strategies. However, little is known about which QI interventions exist for early events of HF after discharge, so the main objective of our study is to conduct a scoping review of the literature to determine which QI strategies are effective for reducing hospital readmissions and mortality for patients with HF who transition from the hospital back into independent living. We will also investigate which elements contribute to effective QI strategies.
\end{abstract}

Methods and analysis: We will search the literature in MEDLINE, EMBASE, CINAHL and the Cochrane library for randomised controlled trials and systematic reviews evaluating QI interventions aimed at improving outcomes for patients with $\mathrm{HF}$ transitioning from the hospital back into the community. Two reviewers will independently apply our eligibility criteria at level 1 (abstract/title) and level 2 (full-text) screening; disagreements will be resolved through consensus. We will extract data in duplicate on study characteristics, population, setting, QI intervention and outcomes. We will synthesise results descriptively and explore QI elements to determine which aspect contributes to its impact. We will also consider synthesis of our data according to several conceptual frameworks such as Wagner's Chronic Care Model.

Discussion and dissemination: The findings of this scoping review will be used to determine which elements should comprise a QI intervention aimed at facilitating the transition of newly admitted patients with HF back into the community. We will disseminate our findings through publications, presentations as well as through a stakeholder meeting to generate key messages most relevant to each.

\section{INTRODUCTION}

Heart failure (HF) is a chronic disease that is associated with substantial morbidity,

\section{Strengths and limitations of this study}

- We will contribute to the knowledge by investigating the specific elements that contribute to the effectiveness of quality improvement (QI) interventions aimed at transitioning patients with heart failure (HF) from the hospital into the community.

- Findings will directly inform the development of a new QI intervention (or adaptation of an existing effective intervention) aimed at improving the care of patients with $\mathrm{HF}$ and reducing rehospitalisations and mortality.

- Many QI interventions are evaluated in nonrandomised trials, so we may not capture all existing QI interventions.

- A limitation of scoping reviews is that the assessment of risk of bias is not part of its conduct since its goal is to provide an overview of the literature regardless of methodological quality.

mortality and utilisation of healthcare resources. It is a leading reason for hospitalisation and readmissions to hospital, particularly among individuals older than 65 years of age. ${ }^{1}$ The costs of HF have been estimated to exceed $\$ 30$ billion in the $\mathrm{USA}^{2}$ and hospital-based care is the major contributor to the direct costs of this condition. HF is also a major healthcare resource and economic burden in Canada, Europe and other developed countries. ${ }^{3}$ Future projections suggest that it will continue to be an important public health concern given our increasingly ageing population. ${ }^{4}$

The prognosis of patients with HF is grim, with high rates of mortality risk and hospital readmissions. ${ }^{1}{ }^{5}$ Within the first year after discharge from hospital, patients on average have a $28 \%$ risk of death, ${ }^{6}$ but despite their high mortality risks, they also exhibit high rates of hospital readmission. ${ }^{4}$ A Canadian 
study found that among patients who survived up to discharge, 24\% were readmitted for HF within 1 year. ${ }^{2}$ Reports from the USA have found that readmissions for any cause occurred in nearly $25 \%$ of patients within 30 days, with the majority of these events occurring within the first 7 days postdischarge. ${ }^{7}$ The transition period early after hospital discharge represents a window of opportunity to positively influence patient outcomes using targeted interventions. ${ }^{8}$ While timely care by healthcare professionals may be an important component of transitional care interventions, ${ }^{9}$ the optimal strategies to reduce readmissions or improve survival among patients with HF are unknown. Adding to the complexity of HF management is multimorbidity, ${ }^{10}$ which makes the care of patients, and implementation of effective interventions and programmes more challenging.

Evidence from systematic reviews suggests that quality improvement (QI) strategies such as multidisciplinary outpatient disease management programmes are beneficial for reducing mortality, and all-cause and HF-specific hospital admissions. ${ }^{5}$ Most of these QI strategies are complex (ie, multifaceted with multiple targets and components). However, few studies have described such interventions and their components in sufficient detail to allow for in-depth and clinically meaningful comparison(s), and it is unknown which components (delivered by whom and to which targets) contribute to their impact. Furthermore, little is known about which QI interventions exist for early events after discharge for inpatients, and no systematic review has previously investigated the impact of QI interventions that focus specifically on optimising the transition of patients with $\mathrm{HF}$ from the hospital to independent living.

The objectives of our study are to conduct a scoping review of the literature for randomised controlled trials (RCTs) and systematic reviews to determine which QI strategies aimed at transitioning adult patients with $\mathrm{HF}$ from the hospital back into independent living are effective for reducing hospital readmissions and mortality. We will also investigate the specific components of QI interventions to identify common elements of those that are effective, and to specifically determine elements that contribute to their effectiveness.

\section{METHODS AND ANALYSIS}

We will use the scoping review methodology as outlined by Arksey and O'Malley ${ }^{11}$ to conduct our study, which is currently considered the most rigorous methodology for conducting scoping reviews. Our protocol was conceived, developed and reviewed by all members of our team.

\section{Eligibility criteria}

We developed our eligibility criteria from our research question: What are effective Quality Improvement strategies for transitioning adult patients admitted to the hospital for $\mathrm{HF}$ back into the community? We used the following PICOS elements to build our eligibility criteria (Population, Intervention, Comparator, Outcomes and Study design): Population: We will include adults (age $\geq 18$ years) who are newly admitted to the hospital or emergency department (ED) with a diagnosis of HF (ie, HF, congestive heart failure (CHF)). We will assume that unless otherwise stated, newly admitted patients are living independently in the community (vs in a long-term care facility). We will exclude patients who are selected or enrolled in the study from outpatient clinics including HF clinics and primary care practice, and those enrolled from administrative databases. Intervention: We will consider any QI intervention aimed at improving outcomes for patients with HF transitioning from the hospital back into the community. We will consider any of: care coordination (a QI strategy involving the deliberate organisation of patient care activities between two or more participants (including the patient) involved in a patient's care to facilitate the appropriate delivery of healthcare services); QI strategies targeting health systems (case management; team changes; electronic patient registry; facilitated relay of information to clinicians; continuous QI); QI strategies targeting healthcare providers (eg, audit and feedback; education; reminders; telemonitoring); and QI strategies targeting patients (patient education; promotion of self-management and reminder systems). Our exclusion criteria are: interventions targeting only healthcare providers or patients unless the intervention included at least one other strategy related to clinician or organisational change; educational strategies focusing on how to educate patients, counselling skills, motivational interviewing, self-directed learning and skills related to the intervention (eg, teaching how to use the website for the RCT); strategies involving ad hoc clinician reminders only; and interventions involving only self-management or reminders unless they also include at least one other strategy related to clinician or organisational change. For example, we would not include an intervention targeted to patients involving reminders alone to monitor glucose. However, if the intervention included case management in addition to reminders to monitor glucose, then we would include it. Comparator: We will consider any standard or usual HF care or control intervention. Outcomes: Our primary outcomes are: hospital or ED readmission; hospitalisation and mortality. Our secondary outcomes are the composite of hospital admission and mortality, hospital length of stay, clinician visits, appropriate use of $\mathrm{HF}$ medications (compliance and adherence) and cost of intervention or costeffectiveness. Outcomes related to adherence or compliance to the intervention or to guidelines rather than HF medications will be excluded. Study design: We will include RCTs, cluster RCTs and systematic reviews for inclusion. Exclusion criteria are: quasi RCTs (non-randomised methods of assignment); studies using other experimental designs (controlled before-after 
studies and interrupted time series); observational studies (prospective/retrospective cohort studies); crosssectional and case-control studies; case reports; and opinion-driven reports (ie, editorials, letters and nonsystematic or narrative reviews). A summary of our inclusion/exclusion criteria are in the online supplementary appendix 1.

\section{Information sources}

We will conduct a systematic search of the literature without language restrictions in MEDLINE, EMBASE, the Cumulative Index to Nursing and Allied Health Literature (CINAHL), and the Cochrane Central Register of Controlled Trials. We will also search unpublished or grey literature from other sources such as healthcare provider organisations (eg, the Canadian Cardiovascular Society, American Heart Association, American College of Cardiology and Heart Failure Society of America). Additionally, we will search the reference lists of included articles and identify other articles through contact with experts in cardiovascular medicine.

\section{Search strategy}

An experienced information specialist developed our search strategy in MEDLINE using the search terms: heart failure, cardiac failure, heart decompensation, myocardial failure; as well as a list of terms for QI strategies. We applied the validated search filters developed by Haynes et $a l^{12}$ to identify RCTs and systematic reviews. ${ }^{13}$ The search strategy was peer reviewed by another experienced information specialist using the PRESS checklist (ie, Peer Review of Electronic Search Strategies). ${ }^{14}$ The search strategy for MEDLINE is available in the online supplementary appendix 2. This was adjusted for our other data sources (EMBASE, CINAHL and the Cochrane Library), which are available from the authors on request.

\section{Study selection}

Our study selection will involve performing an exercise to calibrate reviewers to ensure reliability of screening. Two reviewers will apply our eligibility criteria and independently screen a random sample of 25 citations using our online Synthesi.SR Tool (a proprietary online systematic review software developed for our Knowledge Synthesis Center at St. Michael's Hospital). ${ }^{15}$ We will calculate inter-rater agreement for applying the eligibility criteria (using per cent agreement), and we will repeat this exercise in two subsequent pilot screenings to reach $90 \%$ agreement. Once we attain this level of consistency, two reviewers will independently screen the titles and abstracts of the remainder of potentially relevant articles in duplicate (level 1 screening). We will follow a similar calibration procedure during level 2 screening to identify potentially relevant articles in full text, which will also require two pilot calibration tests to attain a high level of consistency. Disagreements will be resolved through research team consensus for both levels of screening.

\section{Data collection process}

We will develop a standardised data abstraction form in Excel, which will be pilot tested on a random sample of 5-10 included studies to ensure agreement between data extractors. The form will be modified accordingly, and once we reach at least $90 \%$ agreement, data from the remainder of included articles will be extracted independently by two reviewers. We will extract data on study characteristics (eg, first author, citation), population (inclusion criteria), setting, QI intervention and outcomes.

QI interventions are complex (ie, multifaceted with multiple targets), so we will explore their elements to determine which aspects contribute to their impact. To do this, we will extract information about each QI intervention overall, explore its elements and determine which aspects contribute to the intervention's impact. To do this, we will extract information about the overall QI intervention, as well as details about its components or elements (eg, a home-based intervention may include the components such as education, telemonitoring and follow-up with a physician).

\section{Data synthesis}

The analysis will involve collating and summarising all of the findings for each outcome. For RCTs and systematic reviews that do not conduct any statistical testing, results will be presented in a narrative synthesis. Conversely, for studies that include statistical analysis, we will present the statistical estimates (eg, relative risks or HRs, with $95 \%$ CIs) in a tabular format, in addition to summarising them. Interventions determined to be effective by highquality systematic reviews and/or RCTs will be highlighted. We will consider analysing systematic reviews that pool results, and present a summary of findings only for those that do not. We will also prepare descriptive tables to give an overview of the included study characteristics (ie, with the following data presented for all included studies: author, year, target population, study design, intervention vs comparator, outcome(s) studied, follow-up period, etc).

Given that we are conducting a scoping review and not a systematic review, we will not be carrying out a meta-analysis or assessing study quality, as this is not generally performed in scoping reviews. ${ }^{16}$ However, we will consider the synthesis of our data according to several conceptual frameworks such as the Donabedian's structure-process-outcome framework ${ }^{17}$ as well as Wagner's Chronic Care Model (CCM). ${ }^{18} 19$ The aim of CCM is to modify chronic disease care so it becomes patient-centred and high quality. The model provides a structure for organisational/practice change according to six elements for improvement: healthcare organisation, community resources, self-management support, delivery system design, decision support and clinical 
information system. ${ }^{18}$ We will also perform additional and more targeted syntheses on interventions (and their components) identified by our stakeholder team as having potential to inform practice (eg, interventions and their components that are identified as effective and feasible to implement). More specifically, we will use the CCM to map QI interventions and their components according to the six CCM elements to determine which has the most potential for improving the transition of care for patients with HF. As a final step, we will interpret the findings and outline the broader implications for practice and future study.

To explore the elements of QI interventions and determine which of these contribute to their impact, we will use content analysis of the overall QI interventions identified. We will also create taxonomies of $\mathrm{HF}$ QI interventions and their elements and build definitions for each. To do this, two investigators will independently review the description of the overall extracted QI intervention and document its components (eg, telemonitoring, education, prompts) according to who each of the components was delivered by and to which target (eg, education delivered by a study nurse to patients) it was delivered, as well as the frequency and duration of the intervention component (eg, transmission of telemonitored data once per day for 6 months). We will also classify QI interventions into logical categories (eg, disease management interventions). If there are discrepancies between reviewers for documenting this information, we will use group consensus among our team to finalise QI categories, interventions and their components.

\section{Discussion and dissemination}

The findings of this scoping review will be used to determine which elements should comprise a QI intervention aimed at facilitating the transition of newly admitted patients with HF back into the community. In particular, we will identify the specific components of QI interventions that contribute to their impact.

We will use different knowledge translation (KT) strategies to ensure that findings from this scoping review are broadly disseminated to the right audiences. These strategies will include publications in open-access, peerreviewed journals as well as presentation of our work at relevant cardiology and HF conferences (eg, American Heart Association, American College of Cardiology). As part of a more active KT strategy, we will also plan a meeting with our key stakeholders (ie, clinicians, researchers, decision-makers and people with $\mathrm{HF}$ ) to discuss the findings, to generate key messages most relevant to each, and to discuss the next steps including the development of a QI intervention that will address current gaps in care.

Contributors DSL conceived the study. DSL, MK and SS conceived the study design. MK and DSL helped draft the protocol. LP developed and executed the search strategy. All authors edited the draft protocol, and read and approved the final manuscript.

Funding This research was supported through a grant from the Toronto Central Local Health Integration Network. Dr Lee is supported by a clinician-scientist award from the Canadian Institutes of Health Research.

Competing interests None.

Provenance and peer review Not commissioned; externally peer reviewed.

Data sharing statement Unpublished study data such as the search strategies for the other databases (EMBASE, CINAHL, the Cochrane Library) are available on request to the corresponding author.

Open Access This is an Open Access article distributed in accordance with the Creative Commons Attribution Non Commercial (CC BY-NC 4.0) license, which permits others to distribute, remix, adapt, build upon this work noncommercially, and license their derivative works on different terms, provided the original work is properly cited and the use is non-commercial. See: http:// creativecommons.org/licenses/by-nc/4.0/

\section{REFERENCES}

1. Roger VL. Epidemiology of Heart Failure. Circ Res 2013;113:646-59.

2. Go AS, Mozaffarian D, Roger VL, et al. Heart disease and stroke statistics-2014 update: a report from the American Heart Association. Circulation 2014;129:e28-e292.

3. McMurray JJ, Stewart S. Epidemiology, aetiology, and prognosis of heart failure. Heart 2000;83:596-602.

4. Lee DS, Johansen H, Gong Y, et al; for the Canadian Cardiovascular Outcomes Research Team. Regional outcomes of heart failure in Canada. Can J Cardiol 2004;20:599-607.

5. McKelvie RS. Heart failure. BMJ Clin Evid 2011;8:204.

6. Pandor A, Thokala P, Gomersall T, et al. Home telemonitoring or structured telephone support programmes after recent discharge in patients with heart failure: systematic review and economic evaluation. Health Technol Assess 2013;17:1-207.

7. Dharmarajan K, Hsieh AF, Lin Z, et al. Diagnoses and timing of 30-day readmissions after hospitalization for heart failure, acute myocardial infarction, or pneumonia. JAMA 2013;309:355-63.

8. Chun S, Tu JV, Wijeysundera HC, et al. Lifetime analysis of hospitalizations and survival of patients newly admitted with heart failure. Circ Heart Fail 2012;5:414-21.

9. Lee DS, Stukel TA, Austin PC, et al. Improved outcomes with early collaborative care of ambulatory heart failure patients discharged from the emergency department. Circulation 2010;122:1806-14.

10. Liu L, Eisen HJ. Epidemiology of heart failure and scope of the problem. Cardiol Clin 2014;32:1-8.

11. Arksey $\mathrm{H}$, O'Malley L. Scoping studies: towards a methodological framework. Int J Soc Res Methodol 2005;8:19-31.

12. Haynes RB, Wilczynski N, McKibbon KA, et al. Developing optimal search strategies for detecting clinically sound studies in MEDLINE. J Am Med Inform Assoc 1994;6:447-58.

13. Montori VM, Wilczynski NL, Morgan D, et al; for the Hedges Team Optimal search strategies for retrieving systematic reviews from Medline: analytical survey. BMJ 2005;330:68-0.

14. Sampson M, McGowan J, Cogo E, et al. An evidence-based practice guideline for the peer review of electronic search strategies. J Clin Epidemiol 2009;62:944-52.

15. The Joint Program in Knowledge Translation. Synthesi.sr Systematic Review Tool: St. Michael's Hospital. http://knowledgetranslation.ca/ sysrev/login.php

16. Levac D, Colquihoun H, O'Brien KK. Scoping studies: advancing the methodology. Implement Sci 2010;8:19-31.

17. Donabeidan A. Quality assurance. Structure, process and outcome. Nurse Stand 1992;7(11 Suppl QA):4-5.

18. Wagner EH, Austin BT, Davis C, et al. Improving chronic illness care: translating evidence into action. Health Aff (Millwood) 2001;20:64-78.

19. Barr VJ, Robinson S, Marin-Link B, et al. The expanded Chronic Care Model: an integration of concepts and strategies from population health promotion and the Chronic Care Model. Hosp Q 2003;7:73-82. 